\title{
FAST DYNAMIC RECONNECTION AT X-TYPE NEUTRAL POINTS
}

\author{
I. J. D. CRAIG AND P. G. WATSON \\ Department of Mathematics and Statistics, University of Waikato, Private Bag, Hamilton, New Zealand \\ Received 1991 August 7; accepted 1991 December 12
}

\begin{abstract}
We consider the linear and nonlinear evolution of disturbed magnetic X-type neutral points. The problem is formulated within a unified analytic and computational framework which highlights the essence of the magnetic annihilation process, namely, the coupling of a global convection region to a localized diffusion region surrounding the neutral point.

An analytic treatment is given for the case of small disturbances of the equilibrium field in the absence of gas pressure. This problem admits well-defined azimuthal modes which allow a formally exact determination of the magnetic annihilation rate. It is shown that reconnection can only occur in the case of purely radial $(m=0)$ disturbances: the reconnection process is oscillatory and "fast," depending only logarithmically on the plasma resistivity $(\eta)$.

We show that the linear theory supports the notion of an initial implosive stage which rapidly releases the bulk of the energy associated with reconnective field disturbances. This phase is initiated by the advective focusing of the perturbation energy into the neutral point and culminates in the formation of a cylindrical diffusion region of area $A \sim \eta$ and current density $J \sim \eta^{-1}$. This scaling provides a signature for fast linear reconnection.

Next we consider the breakdown of the linear theory. Although fast reconnection is maintained for lowamplitude disturbances in noncylindrical geometries, it is shown that finite gas pressure can stall the reconnection if sufficiently large. This effect, however, may not be critical in more complex X-point geometries. More seriously, for finite-amplitude displacements the cylindrical current structure close to the neutral point is distorted into a quasi-one-dimensional current sheet whose thickness is limited by resistive diffusion. In this case fast reconnection is consistent with a flux pileup solution in which the bulk of the energy is released as heat rather than as the kinetic energy of mass motion.
\end{abstract}

Subject heading: MHD

\section{INTRODUCTION}

It is widely accepted that solar flares are initiated by the rapid collapse of magnetic fields in the low solar corona. As a result, much theoretical effort has gone into investigating mechanisms which can release magnetic energy fast enough to account for the explosive energy release. Early work focused mainly on the magnetic annihiliation that takes place when oppositely directed magnetic fields are pushed together by external "boundary" forces (Sweet 1958; Parker 1963). It was soon recognized, however, that resistive diffusion at localized neutral points must be closely coupled to the global advection of the outer field if the energy release rate is to be sufficient. A crucial advance was provided by Petschek (1964), who demonstrated that field-line reconnection at the origin of an X-type neutral point allows convectively driven magnetic annihilation on time scales approaching the Alfvén time scale of the outer field.

The semiquantitative Petschek analysis has proved highly contentious over the years - claims have been made that the model does not provide a valid solution of the MHD equations--but the mechanism itself appears physically plausible (Vasyliunas 1975; Parker 1979; cf. Biskamp 1986). What seems to be lacking is a dynamically complete evolutionary model of the underlying reconnection process. The problem is that analytic tractability virtually imposes an approximate steady state treatment in which the uniqueness of the solution cannot be guaranteed - hence the different quasi-steady annihilation modes of Petschek (1964) and Sonnerup (1973) (see
Forbes \& Priest 1987). Numerical simulations, on the other hand, are not entirely trouble-free, since a complex diffusionadvection problem in two or more space dimensions has to be modeled (see, e.g., DeLuca \& Craig 1992). One problem is determining the scaling of the annihilation rate given that feasible computations involve unrealistically high plasma resistivities (e.g., the steady state scalings of Biskamp 1986). More critically, because a numerical treatment generally requires assumptions and idealizations that differ from the analytic approach, it is often quite difficult to integrate the disparate, often phenomenological, results of reconnection studies. Priest (1985) implies that the process of magnetic merging seemed quite well understood until numerical simulations came along to cloud the picture! Probably many of the discrepancies in the theoretical picture can be expected (Forbes $\&$ Priest 1987) in view of the differing initial and boundary conditions imposed on the problem.

The purpose of the present paper is to investigate the linear and nonlinear relaxation of a disturbed X-type neutral point configuration. This problem has the advantage of allowing a unified analytic and computational treatment while still containing the essence of the reconnection problem, namely, the coupling of a global convection region to the localized diffusion-reconnection region surrounding the neutral point. Thus, when results can be obtained analytically - for instance, by linearization about the equilibrium solution (see Craig \& McClymont 1991a) - their general validity can be checked using a complete nonlinear treatment. 
As physical motivation, we note that although neutral point topologies will almost certainly be present in the solar corona given the complexity of observed coronal structures, they may be in nonequilibrium as a result of continual buffeting by gasdynamic and MHD disturbances within the active region assemblage. Such disturbances may drive changes in the magnetic field topology that can only be negated by magnetic reconnection in the vicinity of a neutral point.

The relaxation problem is formulated mathematically in $\S 2$. We present a conservative Eulerian formulation of the system that facilitates a convenient semi-implicit numerical treatment. In $\S 3$ an analytic description of the advective field evolution is given, valid for small departures about the equilibrium field; these results are then augmented by numerical resistive solutions and a discussion of the global energy decay. In $\S 4$ the limitations of the linear theory are addressed. Our findings are summarized in $\S 5$.

\section{FORMULATION OF THE PROBLEM}

\subsection{Neutral Point Equilibria}

We imagine an X-type neutral point configuration immersed in a tenuous background plasma of uniform mass density $\rho_{0}$. Coordinates are chosen so that the field strength vanishes at the origin and achieves the value $B_{0}$ at the characteristic radial distance $L_{0}$. We nondimensionalize with respect to $B_{0}$ and $L_{0}$ and write the equilibrium field in the dimensionless form

$$
\boldsymbol{B}_{E}=(y, x) \text {. }
$$

In two-dimensional geometries much simplification is achieved by working with the planar flux function $\psi$ rather than with the magnetic field intensity directly:

$$
B=\nabla \times(\psi / \hat{z})=\nabla \psi \times \hat{z} .
$$

The equilibrium flux function for an $\mathrm{X}$-type neutral point is given by

$$
\psi_{E}=\frac{1}{2}\left(y^{2}-x^{2}\right)=-\frac{1}{2} r^{2} \cos (2 \theta)
$$

in Cartesian and cylindrical coordinates, respectively.

We imagine the equilibrium field to be subject to a spectrum of finite-amplitude disturbances. These may disturb the outer radial boundary to which we assume the field is permanently threaded or line-tied. The perturbed field must then evolve dynamically - reconnecting field lines at the neutral point if the intrinsic topology is not preserved-as it attempts to regain equilibrium. The time scale for establishing equilibrium is measured in units of the boundary Alfvén time scale $\tau_{A}=L_{0} / v_{A}$ $\left[v_{A}=B_{0}\left(4 \pi \rho_{0}\right)^{-1 / 2}\right.$ in Gaussian units], which is typically a few seconds under normal coronal conditions.

To allow a convenient mathematical description, the outer boundary is idealized as a perfect conductor. For linear calculations the assumption of a circular boundary allows perturbations to be represented by azimuthal modes; on the other hand, a rectangular boundary is more suitable for finiteamplitude calculations. Although such boundaries simplify the geometry of photospheric line-tying, they are topologically correct and hence should not distort the underlying physics of the problem.

The key questions concerning the relaxation and resistive energy release of a disturbed X-type neutral point can only be answered by a truly global treatment. We imagine that disturbances in the neutral point configuration are driven in such a way that the flux function is held fixed on the boundaries so

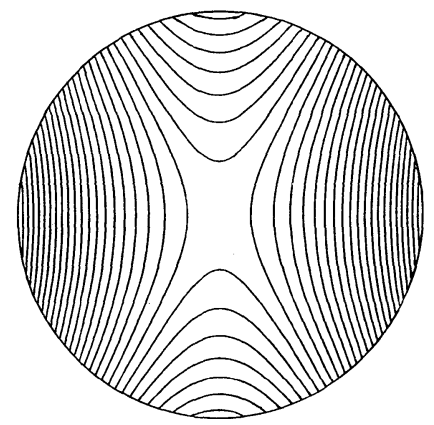

FIG. $1 a$

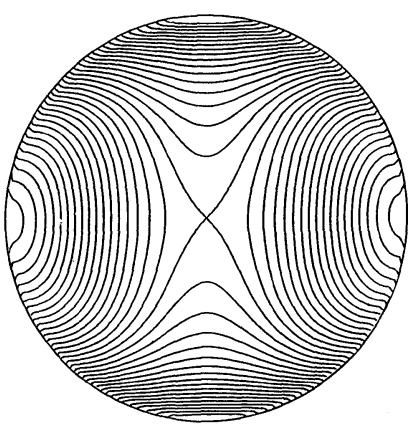

FIG. $1 b$
FIG. 1.-Initial X-point magnetic field structures. In $(a)$ the equilibrium field topology has been altered, and field-line reconnections must occur if the equilibrium is to be achieved; in $(b)$ the original topology has been maintained but reconnections can still occur in the absence of other damping mechanisms.

the initial field is topologically distinct from the neutral point equilibrium, hence field-line reconnections are required to recover the equilibrium. However, we also show in $\S 3$ that reconnections can still occur for initial displacements that do not disturb the intrinsic topology.

Figure 1 illustrates two initial $\mathrm{X}$-point configurations. In Figure $1 a$ the topology of the equilibrium $\mathrm{X}$-point has been changed by the addition of a perturbation that alters the separatrix angle of the field lines; in Figure $1 b$ the field is distorted in a way that maintains the equilibrium topology. Solutions describing the dynamic evolution of these fields are given in $\S 3$.

\subsection{Evolution Equations}

The evolution of the disturbed field is governed by the continuity equation,

$$
\frac{\partial \rho}{\partial t}+\nabla \cdot(\rho v)=0
$$

the momentum equation,

$$
\rho\left(\frac{\partial v}{\partial t}+v \cdot \nabla v\right)=J \times B-\nabla p-v V,
$$

and the induction equation,

$$
\frac{\partial B}{\partial t}=\nabla \times(v \times B)+\eta \nabla^{2} B .
$$

These equations are all nondimensionalized with respect to the far-field boundary values $B_{0}, L_{0}, \tau_{\mathrm{A}}$. The forcing terms in the momentum equation describe, respectively, the Lorentz force, the gas pressure force, and the viscous damping of the plasma. Aside from the effects of finite resistivity, the viscous damping term $(V)$ provides the only mechanism for energy loss in the system. However, in a real plasma the level of viscous damping is expected to be very small if not negligible.

The only other form of dissipation in the system is due to the resistive term in the induction equation. In general, the magnetic Reynolds number is very large $\left[\eta^{-1}>O\left(10^{8}\right)\right]$, which means that the advection of the coronal field dominates everywhere except in the vicinity of a flow stagnation point. The importance of finite resistivity is that it allows field-line reconnections to occur at magnetic neutral points which greatly simplify the global plasma field topology.

Under typical coronal conditions the magnetic pressure dominates the plasma pressure by factors of order 100 or more. 
Accordingly, the Lorentz force is generally the dominant term in the momentum equation (2.4). In terms of the flux function $\psi$ the Lorentz force reduces to

$$
\boldsymbol{J} \times \boldsymbol{B}=-\nabla^{2} \psi \nabla \psi,
$$

where the current density is given by $J=-\nabla^{2} \psi \hat{z}$. Rewriting the induction equation in terms of $\psi$ gives

$$
\frac{\partial \psi}{\partial t}=-v \cdot \nabla \psi+\eta \nabla^{2} \psi
$$

This makes it plain that in the absence of resistive effects the flux function is advected with the flow.

\subsection{Conservative Form of the Evolution Equations}

The field evolution equations are conservative in the absence of the generally small viscous and resistive terms. To bring out this fact, we express the continuity and momentum equations in terms of the divergence of mass and momentum fluxes, respectively, and write the Lorentz force as the divergence of the stress tensor:

$$
T_{i j}=B_{i} B_{j}-\frac{1}{2} B^{2} \delta_{i j}=\frac{1}{2}(\nabla \psi)^{2} \delta_{i j}-\psi_{i} \psi_{j},
$$

where subscripts on the flux function imply differentiation with respect to the subscripted variables. Since the induction equation may be written conservatively in terms of the flux of the quantity $\rho \psi$, we obtain the vectoral form

$$
\frac{\partial \boldsymbol{U}}{\partial t}+\frac{\partial \boldsymbol{F}}{\partial x}+\frac{\partial \boldsymbol{G}}{\partial y}=\boldsymbol{H},
$$

where

$$
\begin{gathered}
\boldsymbol{U}=\left(\begin{array}{c}
\rho \\
\rho u \\
\rho v \\
\rho \psi
\end{array}\right), \quad \boldsymbol{H}=\left(\begin{array}{c}
0 \\
-v V \\
-v W \\
\rho \eta \nabla^{2} \psi
\end{array}\right), \\
\boldsymbol{F}=\left(\begin{array}{c}
\rho u \\
\rho u^{2}+p-\frac{1}{2}\left(\psi_{y}^{2}-\psi_{x}^{2}\right) \\
\rho u v+\psi_{x} \psi_{y} \\
\rho \psi u
\end{array}\right), \quad \boldsymbol{G}=\left(\begin{array}{c}
\rho v \\
\rho u v+\psi_{x} \psi_{y} \\
\rho v^{2}+p-\frac{1}{2}\left(\psi_{x}^{2}-\psi_{y}^{2}\right) \\
\rho \psi v
\end{array}\right),
\end{gathered}
$$

and

$$
\boldsymbol{v}=(u, v), \quad V=(V, W) .
$$

All dissipation terms are represented by components of the vector $\boldsymbol{H}$. Thus, for $\boldsymbol{H}=0$, the problem is entirely advective in nature, and the mass, momentum and $\rho \psi$ are all conserved identically within the volume of the neutral point configuration. The problem of simulating Eulerian advective systems is well studied (Richtmyer \& Morton 1967; Roache 1982), and there is great advantage in retaining the conservative form of the equations. In $\S 4$ we present computational solutions for the disturbed neutral point problem assuming a rectangular outer boundary. For the present we concentrate on an analytic reduction based on a cylindrical formulation of the linearized evolution equations.

\section{THE LINEARIZED PROBLEM}

\subsection{Introduction}

It is clearly unrealistic to expect closed form global analytic solutions for the complete fluid equations. To make analytic progress, we linearize the MHD equations about the neutral point equilibrium neglecting gas pressure and viscous effects. A preliminary discussion of the linearized system, which emphasizes the asymptotic decay rate of the field, is given by Craig \& McClymont (1991a). In this approach it is assumed that the solutions are separable, so that eigenfunctions can be constructed from the resulting complex system of ordinary differential equations. This leads to highly accurate eigensolutions and an analytic description of the reconnective modes, but the difficulty remains of establishing the completeness of the complex radial eigenfunctions (see also Hassam 1991). Thus it is not clear that arbitrary initial conditions can be represented using this approach.

In what follows we take a complementary viewpoint: we regard the linearized problem as defining an initial value rather than an eigenvalue problem; thus the evolution of an arbitrary disturbance is described directly without further assumption. This allows us to explore the validity of the eigensolution method and to investigate more general questions, for instance, the early current buildup prior to reconnection $(\S 3.5)$, and the global energy evolution (§ 3.6).

\subsection{Basic Equations}

To linearize the problem, we set

$$
\psi=\psi_{E}+\delta \psi
$$

and consider only small departures $\delta \psi$ about the equilibrium $\psi_{E}$, given by equation (2.2). The linearized system reduces to

$$
\begin{aligned}
\rho_{0} \dot{v} & =-\nabla^{2} \psi \nabla \psi_{E}, \\
\dot{\psi} & =-v \cdot \nabla \psi_{E}+\eta \nabla^{2} \psi,
\end{aligned}
$$

where for the purposes of this section we identify $\psi$ with the first-order variation of the flux function. Note that we have ignored the equation for the perturbed density, since it can have no influence on the momentum equation in the absence of gas pressure: in effect the fluid is arbitrarily compressible.

Setting the background plasma density $\rho_{0}=1$ and eliminating the explicit dependence on fluid velocity allows the system (3.2) to be written as a single third-order equation for $\psi$ :

$$
\ddot{\psi}=r^{2} \nabla^{2} \psi+\eta \nabla^{2} \dot{\psi} \text {. }
$$

Taking cylindrical modes,

$$
\psi=\psi_{m}\left\{\begin{array}{l}
\sin m \theta, \\
\cos m \theta,
\end{array}\right.
$$

the azimuthal components $\psi_{m}$ satisfy

$$
\ddot{\psi}_{m}=r^{2} \nabla^{2} \psi_{m}+\eta \nabla^{2} \dot{\psi}_{m} .
$$

This equation provides our basic theoretical tool for investigating the linearized system.

\subsection{Reconnection and the $m=0$ Mode}

The interplay between the diffusive and advective aspects of the problem is controlled by the relative magnitude of the terms on the right-hand side of equation (3.5). In the absence of diffusive effects, this equation reduces to $\ddot{\psi}_{m}=r^{2} \nabla^{2} \psi_{m}$, which is simply a magnetic wave equation with wave speed proportional to $r$. Given typical coronal conditions [i.e., $\eta \leq O\left(10^{-8}\right)$ ], such wavelike behavior will extend throughout most of the fluid. Sufficiently close to the neutral point, however, there is always a domain in which diffusion dominates. This domain 
extends from the neutral point out to a critical radius $r_{c}$ which scales as

$$
r_{c}=a \sqrt{\eta}
$$

where $a$ is a number of order unity. Inside this region topological changes are effected as field lines are cut and reconnected at the neutral point. Ignoring advection completely, we obtain

$$
\psi_{m}(r, t)=e^{-\mu_{m} t} J_{m}\left(\sqrt{\frac{\mu_{m}}{\eta}} r\right)
$$

as the solution in the diffusion region. Here $\mu_{m}$ is a constant which can only be determined by global considerations, and the $J_{m}$ are the $m$ th-order Bessel functions. The important point is that the $m=0$ mode is the only mode which allows a finite current - equivalently, a nonvanishing displacement in the flux $\psi$-at the neutral point. It follows that only the $m=0$ component of a disturbance can drive reconnection and hence effect topological change.

\subsection{Boundary Conditions}

The inner boundary conditions on the flux function $\psi_{m}$, namely,

$$
\begin{aligned}
\psi_{m}(0, t) & =0 \quad \text { for } m>0, \\
-\frac{\partial \psi_{m}}{\partial r}-(0, t) & =0 \quad \text { for } m=0,
\end{aligned}
$$

follow immediately from the requirement of a bounded current density at the origin. These conditions also follow from the Bessel function solution (3.7) that holds in the vicinity of the neutral point. On the outer boundary we impose the condition that the perturbed flux $\psi_{m}$ be fixed at some constant value for all time, consistent with line-tying the initial perturbed field. As equation (3.5) is second order in time, we must impose initial conditions on both $\psi_{m}$ and its time derivative. In practice the time derivative is fixed by specifying a zero initial velocity field.

As a precursor to the full treatment of the problem, we first study the initial buildup and localization of the current analytically by neglecting diffusive effects.

\subsection{Analytic Solutions in the Wave Domain}

Analytic solutions can be constructed in the case where the wave zone extends throughout the fluid, i.e., for $\eta=0$. On physical grounds these solutions should provide a good description of the initial evolution of a localized pulse propagating toward the neutral point. For a real resistive plasma, the time for a pulse originating on the outer boundary to reach the edge of the diffusion region $r=r_{c}$ is given by

$$
T=\int_{r_{c}}^{1} \frac{d r}{v} \approx-\frac{1}{2} \ln \eta,
$$

on remembering that the wave velocity $v=r$ and $r_{c}=a \sqrt{\eta}$, with $\eta \ll a$. Hence we expect the ideal solutions derived below to be valid over a time scale comparable to $T$. The fact that $T$ becomes infinite as $\eta \rightarrow 0$ implies that information can never be "advected" into the neutral point. The admission of resistive diffusion into the problem fundamentally changes the picture: the origin is no longer a forbidden point, information from the wave domain can diffuse to the neutral point, so the incoming wave is subject both to resistive attenuation and to reflection from the origin.
Wave solutions are obtained by solving

$$
\ddot{\psi}_{m}=r\left(r \psi_{m}^{\prime}\right)^{\prime}-m^{2} \psi_{m} \text {. }
$$

Introducing the change of variables

$$
s=-\ln r, \quad \varphi_{m}(s, t)=\psi_{m}(r, t),
$$

the wave equation may be rewritten as

$$
\ddot{\varphi}_{m}=\frac{\partial^{2} \varphi_{m}}{\partial s^{2}}-m^{2} \varphi_{m}
$$

In this form we see immediately that an isolated mode given by $\varphi_{m}=\cos (\omega t) \sin (\beta s)$ yields a solution, provided that we take $\omega^{2}=\beta^{2}+m^{2}$. Such modes automatically guarantee the outer boundary condition $\psi_{m}=0$, but the inner boundary condition can be satisfied only by superposing over $\beta$. Thus we obtain the general solution

$$
\psi_{m}(r, t)=\varphi_{m}(s, t)=\int_{0}^{\infty} a(\beta) \sin (\beta s) \cos \left[t\left(\beta^{2}+m^{2}\right)^{1 / 2}\right] d \beta,
$$

where $s=-\ln r$ and from the initial conditions $\psi_{m}=\psi_{m}(r, 0)$, $\dot{\psi}_{m}(r, 0)=0$,

$$
a(\beta)=\frac{2}{\pi} \int_{0}^{\infty} \varphi_{m}(s, 0) \sin (\beta s) d s .
$$

In the important special case of purely $\operatorname{radial}(m=0)$ perturbations, equation (3.13) reduces by virtue of the sine transform, to the usual d'Alembert solution

$$
\psi(r, t)=\frac{1}{2}\left[\varphi^{0}(s-t)+\varphi^{0}(s+t)\right], \quad s=-\ln r,
$$

where we have dropped the $m=0$ subscript and the zero superscript refers to the initial condition, i.e., $\varphi^{0}=\varphi(s, 0)$. Note that $\varphi^{0}$ must be odd to satisfy the outer boundary condition at $s=0$.

To understand the current buildup associated with reconnective modes, it is instructive to explore the d'Alembert solutions in more detail. In general, equation (3.14) implies a separation of an initially stationary localized pulse into outward and inward-propagating components. Once the outward component reflects off the outer boundary, a "pure" inward traveling envelope is formed, say

$$
\psi(r, t)=H(t-\ln r) .
$$

Two interesting results follow from this equation. First, from the induction equation $|\boldsymbol{v}|=|\dot{\psi} / \boldsymbol{r}|$, and so $|\boldsymbol{v}|=|\delta \boldsymbol{B}|=$ $|\partial \psi / \partial r|$. Hence there is a local and global equipartition of magnetic and fluid energies with $v$ given by

$$
v=-H^{\prime} \nabla \psi_{E} / r^{2},
$$

where the prime denotes differentiation with respect to the function argument and we have used the fact that velocities can develop only in the direction of the Lorentz force. Second, we note that the envelope travels toward the neutral point retaining its amplitude, but steepening because the back of the pulse travels faster than the leading edge. This means that very large localized currents must form as the wave approaches the diffusion region.

Let us consider two concrete examples of the implosive current buildup: first, we take a global disturbance that contaminates the neutral point and hence requires reconnection to damp out; second, we consider a localized pulse in the outer 
field that maintains the intrinsic topology of the field equilibrium. These initial magnetic configurations are sketched in Figure 1. In the first case,

$$
\psi^{0}=k\left(1-r^{2}\right),
$$

so that $\psi(0)=k, \psi(1)=0$. This corresponds to the closing up of the orthogonal $X$ to an $X$ which makes an angle $\alpha$ with the $x$-axis $(\alpha<\pi / 4)$, as can be seen by the identification $k=$ $-\frac{1}{2} \cos (2 \alpha)$. To satisfy the out er boundary condition at $s=0$, we require that $\varphi^{0}$ satisfy $\varphi^{0}(-z)=-\varphi^{0}(z)$. Substituting $\varphi^{0}=k\left(1-e^{-2 s}\right)$ in equation (3.14), we find

$$
\psi(r, t)= \begin{cases}k\left[1-r^{2} \cosh (2 t)\right] & \text { if }-\ln r>t, \\ k e^{-2 t} \sinh (-2 \ln r) & \text { if }-\ln r<t .\end{cases}
$$

Next we consider the solution for a pulse initially localized in the outer field. Specifically, we take

$$
\varphi^{0}=s e^{-s^{2}}
$$

and construct the d'Alembert solution by replacing $s$ with $s \pm t$.

It should be stressed that the advective solution will always provide a good description of the initial development of the resistive solution, provided $\eta$ is sufficiently small. Resistive solutions for each of the initial conditions considered above are discussed in the next section. The key point is that the advective motion of a radial magnetic wave toward the origin always drives a massive current buildup that initiates reconnection. This initial development-namely, the constant-amplitude advection of $\psi$ toward the origin-is illustrated in Figures 2 and 3 discussed below.

\subsection{Simulations of the Resistive Fluid}

The admission of finite resistivity into the problem means that information from the wave zone can now diffuse to the origin. We can think of diffusion becoming the dominant process when the diffusive speed $v_{D}$ exceeds the wave speed (which scales as $r$ ). On dimensional grounds this occurs when

$$
v_{D} \approx \frac{\eta}{r} \approx r
$$

and so information will cross the diffusion region in unit time. Thus a localized pulse originating at the outer boundary reaches the origin on a time scale given by $1+T$, where $T$ is given by equation (3.9). Hence, for small enough $\eta$ the "communication time" between the origin and the outer boundary scales as $\ln \eta$.

In simulating equation (3.5) for finite $\eta$, it is convenient to work in the $s=-\ln r$ variable. Thus we write equation (3.5) in the form

$$
\ddot{\varphi}_{m}=\nabla^{2} \varphi_{m}+\eta e^{2 s} \nabla^{2} \dot{\varphi}_{m}
$$

where

$$
\nabla^{2} \varphi_{m}=\frac{\partial^{2} \varphi_{m}}{\partial s^{2}}-m^{2} \varphi_{m}
$$

In practical simulations we truncate the infinite interval $s_{\max }=\infty$ by taking $s_{\max }$ to be some small fraction of $\ln r_{c}$, with $r_{c}$ defined as in equation (3.6).

Figures 2 and 3 show the resistive attenuation of the solutions for the initial conditions (3.15) and (3.17) discussed above, with $\eta=10^{-8}$. Each plot displays sequential time slices of the flux function and the current over roughly 30 Alfvén times. The initial advective development in both figures is extremely well described by the d'Alembert solution. As predicted, the localized pulse initially breaks up into separate inward- and outward-moving waves, but once the outgoing wave reflects off the outer wall, a pure ingoing pulse is formed which advects toward the origin. The pulse amplitude decays markedly only on entering the diffusion region-which occurs after some 8 Alfvén times, in accordance with equation (3.9). Far more spectacular is the initial buildup of current brought about by the localization of the wave: the maximum current amplitude in fact scales inversely with $\eta$ (as discussed in $\S 4.1$ below). In both solutions the bulk of the energy associated with the disturbance is annihilat ed during the initial implosive phase.

What are the decay times associated with the resistive energy dissipation? To answer this question, we compute the evolution of the global (magnetic plus fluid) energy of the disturbance using the scheme discussed in the Appendix. Figures 4 and 5 show time plots of the total energy for the resistive solutions under discussion. Both decay curves reflect oscillatory behavior on the $\ln \eta$ time scale as predicted. But the most prominent feat ure is the steplike decay of the total energy, especially during the first few bounce times. For large $t$ there is a relatively slow, mean asymptot ic decay rate with an underlying ripple structure (again with the $\ln \eta$ dependence). This structure is real and can be explained by the fact that the magnetic and fluid energies are never exactly out of phase for finite $\eta$.

\subsection{Eigenfunction Description of Reconnective Modes}

It is interesting to compare the present results with the eigenfunction approach of Craig \& McClymont (1991a), which assumes solutions of the form

$$
\psi_{m}(r, t)=\operatorname{Re}\left[f(r) e^{\lambda t}\right],
$$

where $f(r)$ is complex and oscillation and decay rates are given by

$$
\lambda=-\alpha+i \omega \text {. }
$$

Craig \& McClymont show that for sufficiently small $\eta$ reconnective $(m=0)$ solutions can be constructed by matching the inner diffusive solution of equation (3.7) to "standing waves" in the outer field of the form $\sin (\beta \ln r)$, as suggested by equation (3.13). In particular, the oscillation and decay rates are given by

$$
\omega=-\frac{(2 n+1) \pi}{\ln \eta}, \quad \alpha=\frac{\omega^{2}}{2(n+1)},
$$

respectively, where $n$ is the number of radial nodes. Thus the decay rate and oscillation frequency are limited by the fundamental $n=0$ mode, which has no radial crossings. Perhaps not surprisingly, the decay rate we compute and the radial form of the simulated solutions are perfectly described by the fundamental eigenmode in the limit of large $t$. Indeed, from the present "causal" viewpoint the fundamental frequency can be identified with the "signal travel time" (eq. [3.9]). An important point physically is that the oscillation and decay rates are "fast," depending only logarithmically on the resistivity.

At first sight it appears that the eigenfunction model does not provide a good description of the initial evolution, especially the first localization and diffusion cycle in which the bulk of the energy is lost (see Figs. 4 and 5). The fundamental eigen- 


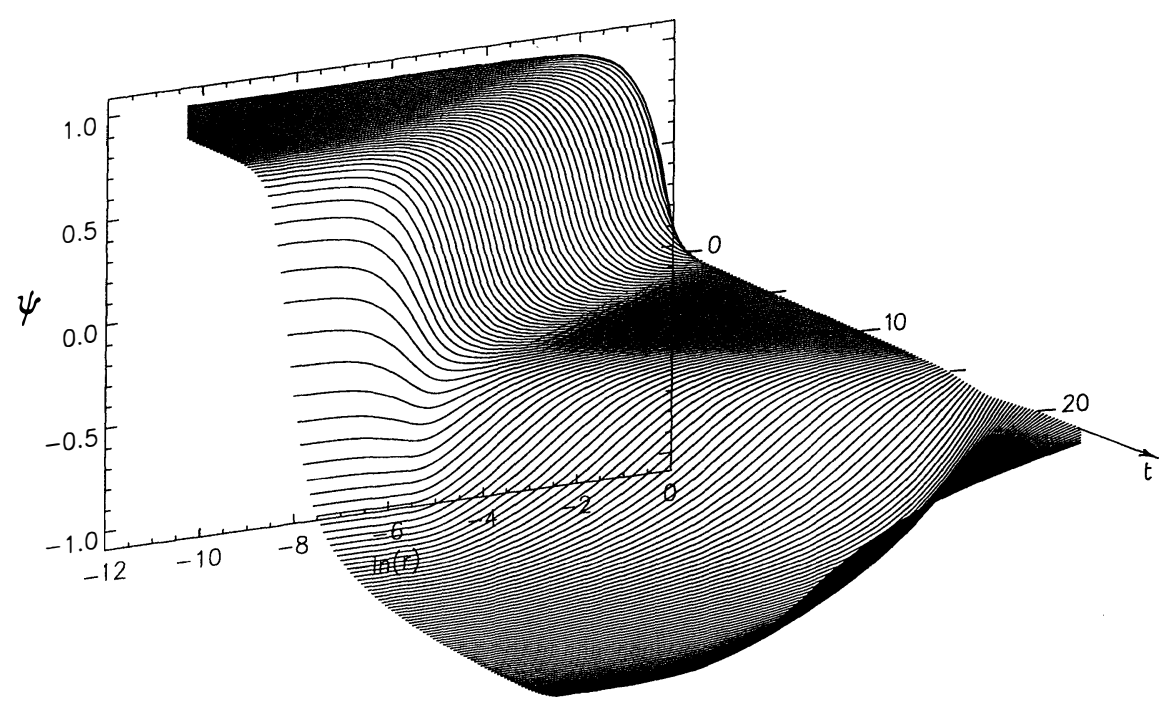

FIG. $2 a$

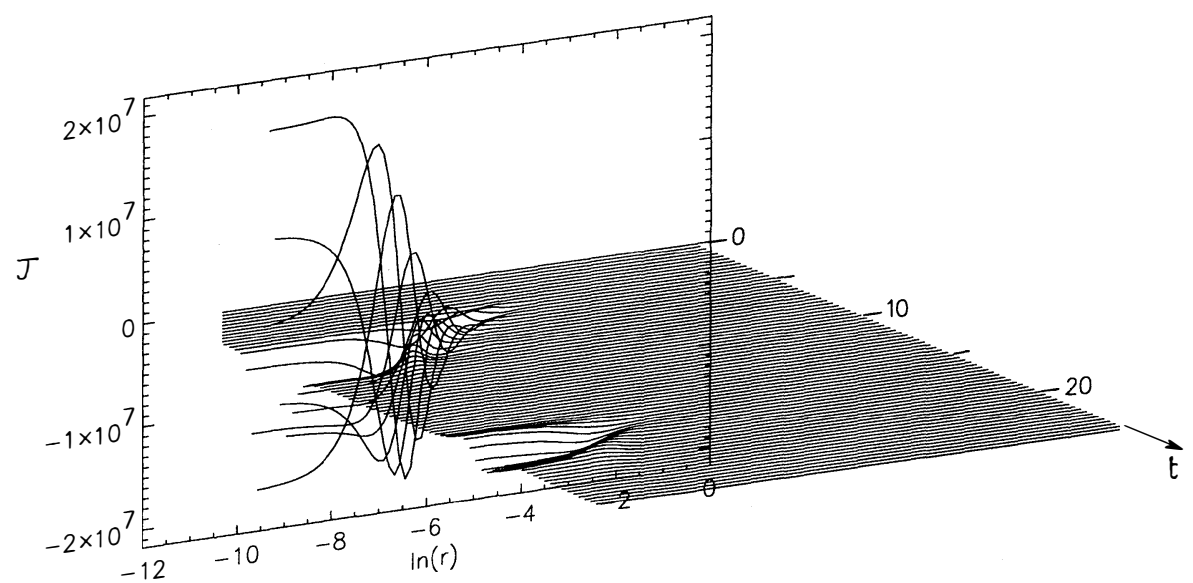

FIG. $2 b$

FIG. 2- (a) Time-slice diagram of the evolving flux function assuming $\psi^{0}=k\left(1-r^{2}\right)$ as the initial condition (with $k=1$ ). The time slices are evenly spaced out to

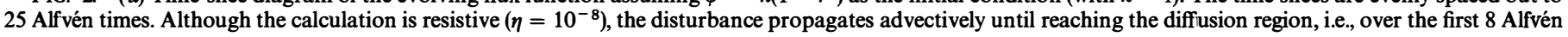
times. (b) Time-slice diagram of the current density.

function only becomes accurate at large $t$, once the disturbance has been globalized by diffusion. However, detailed independent calculations (Craig \& McClymont 1991b), which represent a pulse as a superposition of higher $n$ nodes, can mimic both the steplike energy decay and the rapid initial energy loss that precedes the slower asymptotic decline. The presence of rapidly decaying higher nodal contributions implied by an initially localized wave means that, although the fundamental decay rate is universally applicable at large $t$, it generally underestimates the reconnection rate during the first few oscillation cycles.

\subsection{Higher $m$ Modes}

Higher azimuthal modes are not associated with reconnection. Thus, in contrast to the fundamental radial modes, it is possible for higher modes to decay by nonresistive mechanisms - for instance, damped fluid motions. It is not our present intention to provide a detailed discussion of nonreconnective disturbances. We simply point out that higher azimuthal disturbances are characterized by disturbances in the outer field, rather than in the vicinity of the neutral point. Thus, although the presence of added azimuthal nodes increases the oscillation frequency and decreases the wavelength of the disturbance which we would expect to increase the decay rate, this is counterbalanced by the absence of a strong localization in the vicinity of the neutral point. Craig $\&$ McClymont (1991b) show that for moderate $m$ the oscillation frequency and damping rate are given by

$$
\omega^{2}=\beta^{2}+m^{2}, \quad \alpha=\frac{\beta^{3}}{4 m(n+1)},
$$

where

$$
\beta=-\frac{(n+1) 2 \pi}{\ln \eta}
$$

The decay rates are reduced slightly below the reconnective rate, but a logarithmic dependency of the solution is retained. 
No. 1,1992

DYNAMIC RECONNECTION AT X-TYPE NEUTRAL POINTS

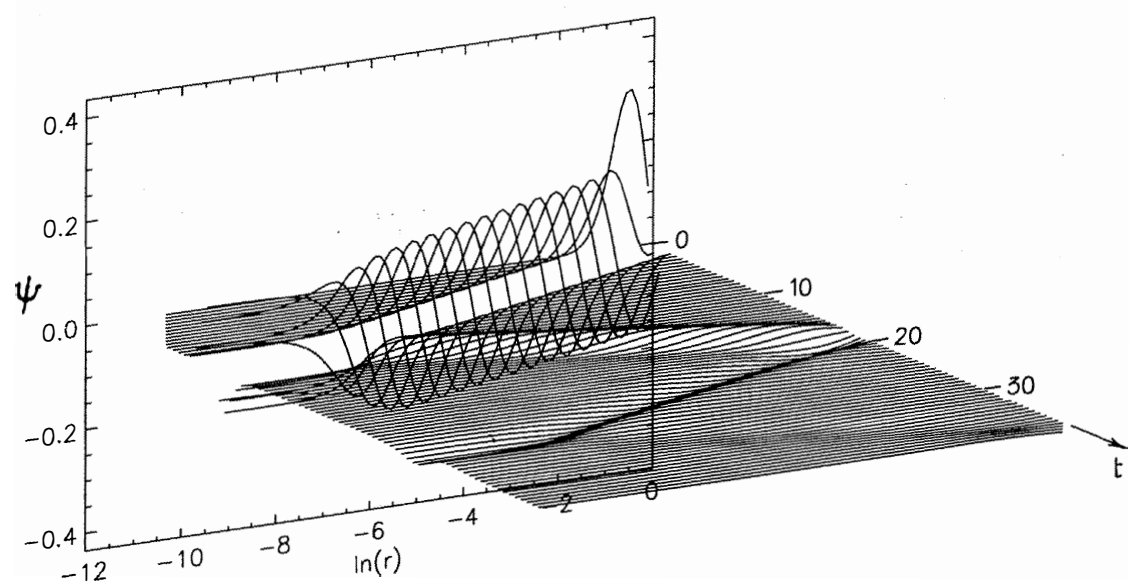

Fig. $3 a$

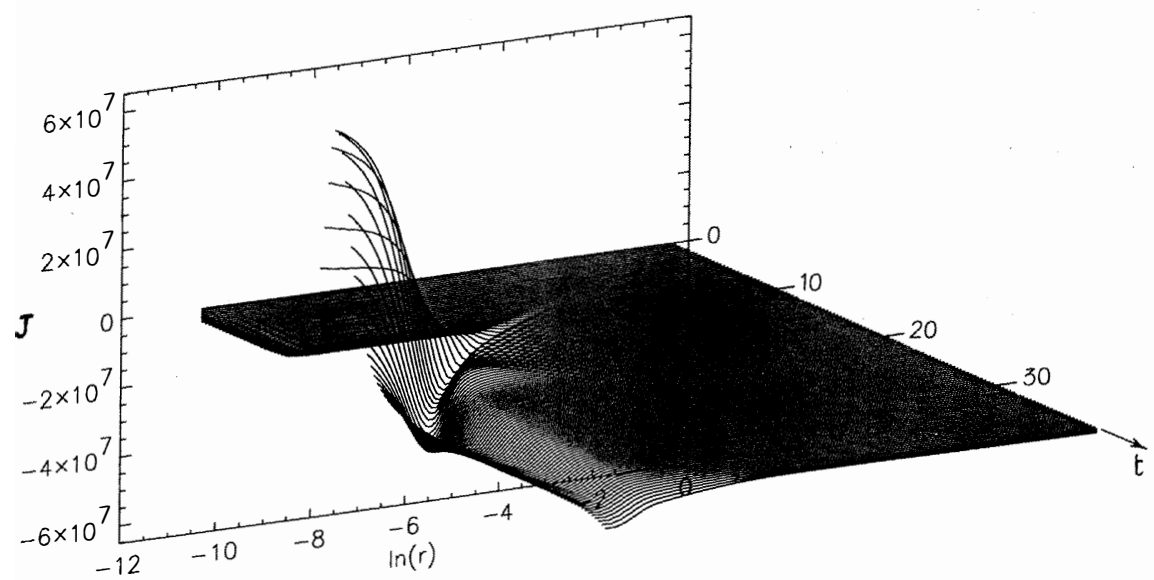

FIG. $3 b$

Fig. 3. $-(a)$ Time-slice diagram of the evolving flux function assuming $\psi^{0}=s e^{-s^{2}}$ as the initial condition with $\eta=10^{-8}$. The time axis runs to 37.5 Alfvén times In contrast to the initial condition of Fig. 2, this pulse disturbance does not change the intrinsic topology of the neutral point. Nonetheless large currents are driven at the neutral point - the signature for topological reconnection-as shown in $(b)$.

\section{LIMITATIONS OF THE LINEAR THEORY}

\subsection{Scaling Laws in the Linear Model}

We have seen that the linearized theory predicts that fast reconnection should occur naturally, even for small perturbations: it does not ha ve to be driven continuously by bound-

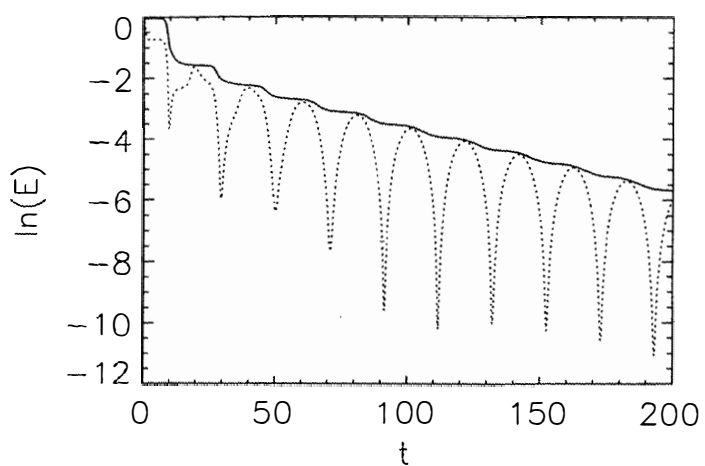

Fig. 4.-Plot of the log of the total energy vs. time for the run of Fig. 2. The dotted line shows the magnetic energy. Note the steplike behavior of the energy decay and the gradual descent into the asymptotic solution described by the fundamental radial mode. ary disturbances contrary to the implications of steady state models. Also, the reconnective mode is os cillatory in natu re: the fundamental oscillation frequency is determined by the signal travel time from the outer boundary to the diffusion region and depends only logarithmically on $\eta$. But although the as ymptotic decay rate is determined by the decay of the

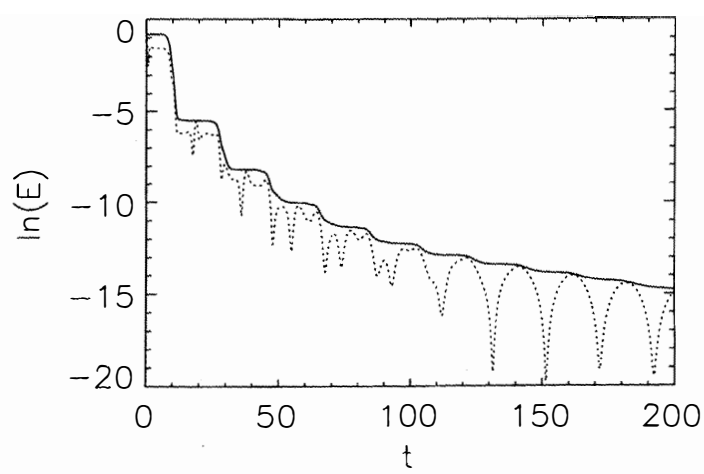

Fig. 5.-Plot of the log of the total energy vs. time for the run of Fig. 3. The steplike nature of this plot is more pronounced than for Fig. 4, owing to the stronger localization of the initial disturbance.

Data System 
fundamental reconnective mode (eq. [3.20]), the bulk of the perturbation energy is lost very quickly during the initial implosive phase.

Let us consider the implosion in more detail. In this phase the energy of a reconnective perturbation becomes focused into an increasingly localized cylindrical current spike, while outside this region the configuration is current-free. This fact allows us to deduce key properties of linear reconnection on the basis of simple energetic arguments. At any stage in the advective collapse we can write the perturbation energy in terms of the excess magnetic and kinetic energy of the fluid:

$$
\delta E=\frac{1}{2}\left\langle\delta B^{2}\right\rangle+\frac{1}{2}\left\langle\rho v^{2}\right\rangle,
$$

where $\delta B$ is the magnitude of the perturbed field, and the angle brackets (see the Appendix) denote integration over space. Initially all the excess energy is magnetic, but as the current localizes the fluid energy increases until equipartition is achieved (i.e., $\delta E=\left\langle\delta B^{2}\right\rangle$ ). The current localization is limited only by resistive diffusion close to the neutral point. If $A$ is the area enclosing the cylindrical wave, then

$$
\left\langle\delta B^{2}\right\rangle=\delta E \approx \delta B^{2} A
$$

is invariant during the phase of current buildup. Since $A \approx \pi R^{2} \approx \pi \eta$ and $\delta B \approx J R$, we deduce that the scalings

$$
J \sim \frac{1}{\eta}, \quad A \sim \eta
$$

should be valid at the time of maximum current buildup. In this case both the ohmic dissipation rate and the flux annihilation at the neutral point scale independently of any power of $\eta$ :

$$
W_{\eta}=\eta J^{2} A \sim \eta^{0}, \quad \dot{\psi}=\eta J \sim \eta^{0} .
$$

Likewise, the total current enclosed by the cylindrical wave is invariant. We can take these scalings as a signature for fast linear reconnection during the implosive phase.

When does the linear theory break down? The requirement that the perturbed field remain less than the background field, imposes the condition

$$
|\nabla \psi|<\left|\nabla \psi_{E}\right|=r .
$$

Since the advective solution holds only for $r^{2}>\eta$, we have that the initial perturbation amplitude must satisfy $\psi<O(\eta)$ to maintain linearity. That this condition is highly restrictive is a manifestation of the rapid buildup of the perturbed field close to the neutral point. We discuss the influence of nonlinear effects in $\S 4.3$ below. For the moment we consider the idealizations of cylindrical geometry and arbitrary compressibility within the context of small-amplitude displacements.

\subsection{Compressible Plasmas in Noncylindrical Geometries}

To investigate whether fast reconnection persists under more general conditions, we first break the azimuthal symmetry by replacing the circular outer boundary by a square superconductor, but retain the assumption of small-amplitude reconnective disturbances. Results are obtained via an alternating direction implicit solution (see Craig \& Sneyd 1990) of the resistive induction equation in system (2.8). We also briefly consider the influence of an adiabatic gas pressure term in the momentum equation.

In the case of zero gas pressure $(\beta=0)$, it is always possible to represent an initial perturbation as a superposition of low- order azimuthal disturbances, viz.,

$$
\psi=\sum_{m} f_{m}(r) e^{i m \theta} .
$$

This suggests that the fast reconnective scaling will be obeyed provided only that the initial displacement contains a significant $m=0$ component. Figure 6 confirms that the fast scaling is retained. For $\beta=0$ both the maximum current (over space and time) and the area over which the current is distributed (defined as the area for which the current density remains within a factor of three of the spatial maximum) scale according to the fast prediction. Since the asymptotic time dependence of the solution also displays the oscillatory time development of the linear model, it is clear that simulations involving rectangular boundaries are completely compatible with the cylindrically symmetric theory.

It is not true, however, that fast reconnection is independent of the gas pressure. We find that even small plasma pressures can wash out the asymptotic oscillatory reconnection phase (the large- $t$ behavior of Craig \& McClymont 1991a and Hassam 1991). More critically, the initial implosion is weakened if the gas pressure is sufficiently high: this can be anticipated theoretically by noting that $\beta \nabla \rho$ must scale as $\beta / r^{2}$ times the Lorentz force according to solution (3.15). Included in Figure 6 are the scalings of current and area obtained by assuming adiabatic pressure of amplitude $\beta=0.1$. The total current enclosed by the wave is again invariant, but the current density increases too slowly with decreasing $\eta$ to be consistent with fast reconnection. The reconnection stalls because the gas

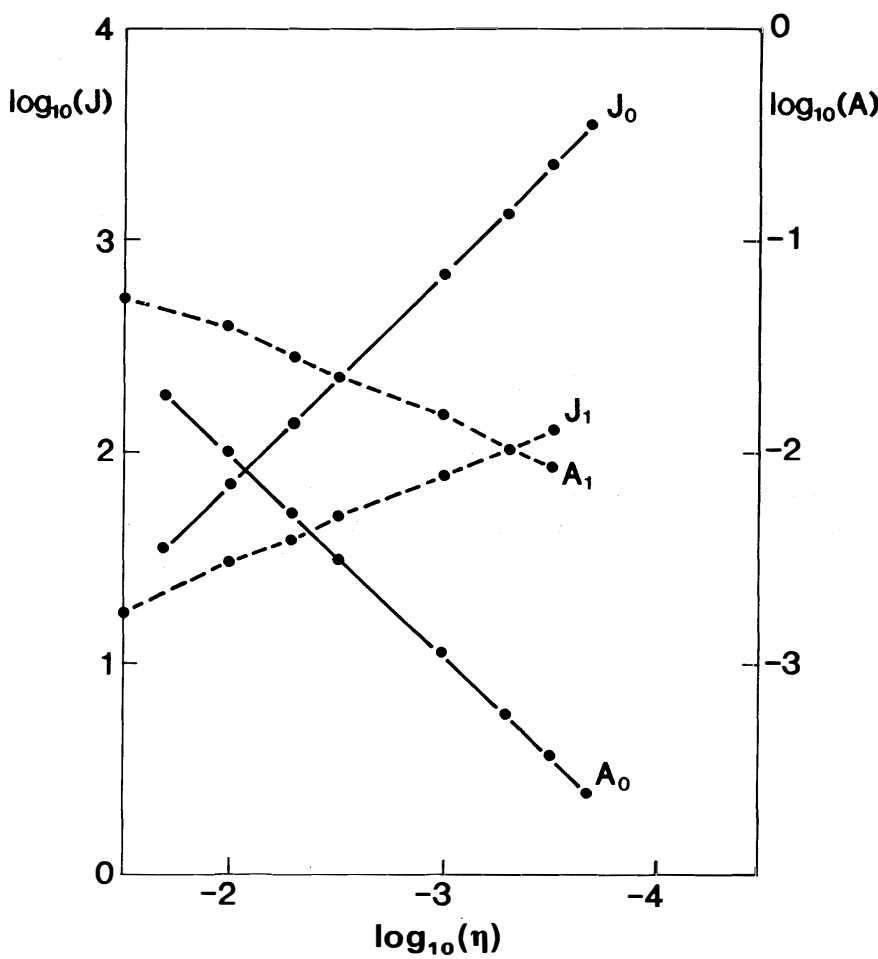

FIG. 6.-Plot of the current and area scaling as a function of $\eta$. The fast reconnective scaling predicted by linear cylindrical theory is obtained in rectangular geometry in the case of zero gas pressure and small amplitude disturbances (solid lines). For gas pressures of order $\beta=0.1$, the fast scaling breaks down: although the total current enclosed by the cylindrical wave remains constant, the wave area can no longer localize strongly enough to maintain fast reconnection. 
pressure eventually builds up close to the neutral point opposing the impulsive collapse. Still higher gas pressures reduce the reconnection rate more dramatically and lead to static diffusion rates that scale as $\eta$. This result also holds good for large-amplitude disturbances.

We cannot, however, use the present results as evidence against fast reconnection in general. It appears that gas pressure effects are quite dependent on the complexities of the flow topology, for there is some evidence that two-dimensional, quasi-periodic, closed $\mathrm{X}$-point topologies may retain fast reconnective scaling even for incompressible fluids (Biskamp \& Welter 1980; DeLuca \& Craig 1992). The stalling of fast reconnection in the present case could then arise from the overly restricted flow pattern imposed by the simple closed geometry.

\subsection{Nonlinear Effects}

In what follows, we present an argument that unifies the present analysis with the classical picture of the quasi-onedimensional neutral sheet. Although our arguments are mainly schematic, they are well supported by the detailed solutions of system (2.8) as well as independent nonlinear simulations (DeLuca \& Craig 1992; Craig \& McClymont 1991b).

In general, a reconnective disturbance of the X-point [with $\psi>O(\eta)]$ will become nonlinear at some point during the initial implosion, well before the diffusion phase sets in. As the disturbance focuses toward the origin, the perturbed field builds up in amplitude and begins to interact with the background field, causing cancellation and reinforcement in adjacent lobes of the X-point. Accordingly, the imploding wave loses cylindrical symmetry and becomes quasi-one-dimensional as it stalls/accelerates in regions of weak/strong magnetic field. The end result, as illustrated in Figure 7 , is the emergence of a classical current sheet! Thus the current-sheet morphology changes from a localized "spike" to a quasi-rectangular "tombsto ne" as the problem becomes nonlinear.

The radius at which the cylindrical wave becomes nonlinear can be estimated from equation (4.2). Setting $\delta B=B_{E}=R_{c}$ gives

$$
\pi R_{c}^{4} \approx \delta E
$$

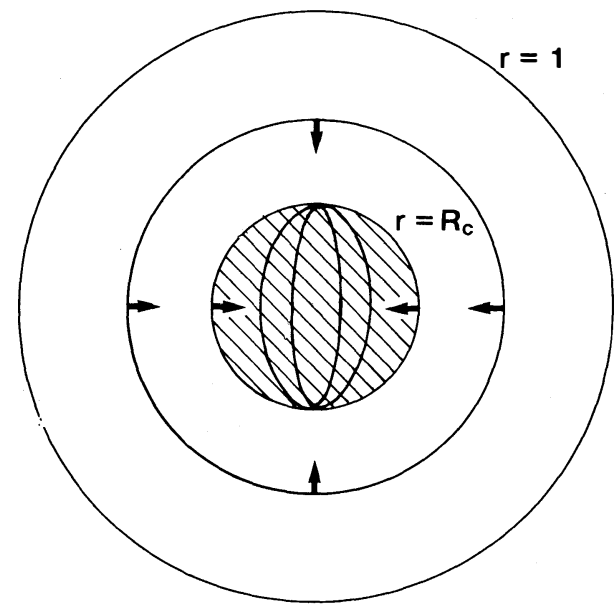

Fig. 7.-Sketch of the breakdown of cylindrical symmetry close to the neutral point due to finite-amplitude disturbances. An incoming quasicylindrical wave is distorted into a quasi-one-dimensional wave inside $\boldsymbol{R}_{c}$. Resistive diffusion eventually limits the thickness of the resulting current sheet. which shows that $R_{c}$ scales as $(\delta E)^{1 / 4}$. Inside $R_{c}$ our cylindrical formulation breaks down in the manner of Figure 7. Equation (4.1), however, remains valid in the quasi-one-dimensional collapse until resisti ve effects take over. Equation (4.2) also applies if we interpret $\delta E$ as the (invariant) excess magnetic energy associated with neutral point currents in the limit of small $\eta$. Writing equation (4.2) in the form

$$
\delta B^{2} L l \approx \delta E,
$$

we identify the length $L$ of the current sheet with $R_{c}$. Since $R_{c}$ is invariant for a fixed perturbation, it is the variation of the sheet thickness $l$ with $\eta$ that determines the scaling of the monlinear reconnection: the thinner the sheet, the faster the magnetic annihilation.

A simple description of the quasi-one-dimensional collapse can be obtained by replacing the cylindrical wave equation by a one-dimensional model in which the wave speed scales as the linear distance from the neutral line. This model is conservative, since it underestimates the nonlinear advective speed: for the one-dimensional ideal collapse to singularity occurs in finite rather than infinite time (Imshennik \& Syrovatskii 1967; Forbes \& Speiser 1979). Equating the diffusion and advection speeds then gives the skin depth formula $l \approx \eta^{1 / 2}$. This leads to the scaling

$$
J \sim \eta^{-3 / 4}, \quad A \sim \eta^{1 / 2}
$$

and

$$
W_{\eta} \sim \eta^{0}, \quad \dot{\psi} \sim \eta^{1 / 4} .
$$

This is a flux pileup solution - the magnetic field at the edge of the sheet scales as $\eta^{-1 / 4}$ in which the bulk of the excess energy appears as heat rather than kinetic energy of mass motion (see DeLuca \& Craig 1992). More detailed considerations of the one-dimensional collapse (at least in the absence of gas pressure; see Bulanov \& Ol'shanetskii 1985 or Sakai 1990) tend to enhance rather than diminish the reconnection rate. Either way, it appears to be a feature of the nonlinear collapse that flux pileup is required to maintain the fast dynamic reconnective scaling.

\section{CONCLUSIONS}

We have considered the time evolution of a disturbed X-t ype neutral point. The evolution of the plasma is determined by the coupling of large-scale advective motions in the outer field to localized magnetic annihilation in the vicinity of the stagnation point. In particular, disturbances which upset the intrinsic topology of the equilibrium field-for instance, by narrowing the $\mathrm{X}$ - can relax only by driving field-line reconnections at the origin. However, disturbances which maintain the equilibrium topology can also cause field-line reconnections to occur provided that other damping mechanisms are weak enough.

We have formulated the relaxation problem for both smalland large-amplitude disturbances. For sufficient ly small perturbations the localized current structures which develop at the neutral point remain cylindrical, but for larger amplitude disturbances cylindrical symmetry is lost and quasi-onedimensional sheets are formed. In either case, the cylindrical model provides a graphic description of the initial implosi ve collapse of the disturbance toward the neutral point.

We have shown that the linearized problem admits welldefined azimuthal modes, with only the $m=0$ mode corresponding to topological reconnection. A universal feature of 
the problem is that small disturbances in the outer field drive wave motions which strongly localize near the origin, generating large currents: thus incoming waves are resistively attenuated as they traverse the diffusion region prior to reflection from the stagnation point. In this way, advective motions of the outer field drive fast oscillatory reconnection with a fundamental frequency $\omega=-\pi / \ln \eta$ and asymptotic decay rate $\omega^{2} / 2$.

Perhaps of greater physical significance is the fact that the bulk of the perturbation energy is lost as a result of the initial implosion prior to the asymptotic decay. This can be understood in terms of the very fast decay of higher nodal $(n>0)$ components in the eigenfunction representation of the initial conditions. Yet fast linear reconnection is not restricted to cylindrical geometries: Cartesian formulations maintain all the characteristics of fast reconnection, in particular the scalings of current density and current-sheet area (as discussed in $\S 4$ ). The effects of finite gas pressure, however, wash out the oscillatory reconnection phase and, if sufficiently large, can stall-at least in the present geometry-the initial implosive energy release. This result also holds good for large-amplitude disturbances, but its physical significance is compromised by the fact that more complex magnetic flow topologies may allow fast implosive reconnection to persist even for incompressible plasmas (DeLuca \& Craig 1992).

In $\S 5$ we considered the breakdown of the linear theory. In general, a low-amplitude topological disturbance is manifested as an inward-propagating cylindrical wave that gradually steepens and eventually becomes nonlinear at some radius $R_{c}$ determined by the energy of the perturbation. We can think of the neutral point focusing the perturbation energy toward the origin. The outer field is left current-free, but inside $R_{c}$ the perturbation energy becomes increasingly localized into a quasi-one-dimensional current sheet whose thickness is limited by resistive diffusion. The current structure now resembles a rectangular "tombstone" rather than the cylindrical "spike" of the linear theory. This change in morphology means that the magnetic flux now has to pile up at the edge of the sheet in order to maintain fast reconnection: in this case the length of the sheet, though ultimately limited by the global geometry, depends mainly on the energy of the perturbation (via eq. [4.4]), whereas the sheet thickness scales as $\eta^{1 / 2}$ or faster. In such cases the perturbation energy is mainly converted to heat via Ohmic dissipation rather than to the kinetic energy of mass motion. DeLuca \& Craig (1992) provide concrete examples of dynamic flux pileup solutions.

Frequent discussions with Sandy McClymont and Franklin Sneyd have been greatly appreciated. We would also like to thank Graham Rickard and Mark Billinghurst for computational assistance and Terry Forbes for comments on an early version of the manuscript.

\section{APPENDIX}

\section{GLOBAL ENERGY CALCULATION}

The fact that the zeroth-order field is a magnetic equilibrium implies that the first-order energy variations must vanish. This means that the excess energy of the linearized system is given by the second-order variations of magnetic and kinetic energy, $\delta^{2} M$ and $\delta^{2} K$, respectively. The kinetic energy variation is given by

$$
\delta^{2} K=\left\langle\frac{1}{2} v^{2}\right\rangle,
$$

where $\langle\ldots\rangle=\int \ldots d V$, with $d V=r d r d \theta$ in cylindrical polars. The magnetic energy variation initially appears more complicated:

$$
\delta^{2} M=\frac{1}{2}\langle\delta B \cdot \delta B\rangle+\left\langle\delta^{2} B \cdot B_{E}\right\rangle
$$

but recasting this equation in the form

$$
\delta^{2} M=\frac{1}{2}\langle\delta B \cdot \delta B\rangle-\frac{1}{2}\left\langle\delta B \cdot\left(\xi \times J_{\bar{E}}\right)\right\rangle
$$

by introducing the fluid displacement $\xi$ shows that the second-order contribution $\left\langle\delta^{2} \boldsymbol{B} \cdot \boldsymbol{B}_{\boldsymbol{E}}\right\rangle$ vanishes, since the equilibrium current $J_{E}$ is zero. Hence we can write the change in global energy in the simple form

$$
\delta^{2} U=\frac{1}{2}\langle\delta B \cdot \delta B\rangle+\frac{1}{2}\left\langle v^{2}\right\rangle .
$$

To calculate the fluid energy, we first note, from the momentum equation (3.2), that any velocity increment $d v$ is in the direction of $\nabla \psi_{E}$, which is perpendicular to the magnetic field lines. This allows us to write $v(r, \theta, t)=v_{m}(r, t) \cos m \theta(\nabla \psi /|\nabla \psi|)$, and we can compute the velocity magnitude from the induction equation via

$$
v_{m}(r, t)=\frac{1}{r}\left(\eta \nabla^{2} \psi_{m}-\frac{\partial \psi_{m}}{\partial t}\right)
$$

Thus a simple space quadrature over the flux function and its derivatives suffices to compute the global energy variation

$$
\delta^{2} U=\frac{1}{2} \int\left[\left(\nabla \psi_{m}\right)^{2}+v_{m}^{2}\right] d V
$$


Biskamp, D. 1986, Phys. Fluids, 29, 1520

Biskamp, D., \& Welter, H. 1980, Phys. Rev. Lett., 44, 1069

Bulanov, S. V., \& Ol'shanetskii, M. A. 1985, Soviet J. Plasma Phys., 11, 425

II Craig, I. J. D., \& McClymont, A. N. 1991a, ApJ, 371, L41

I $\rightarrow$ I $-1991 \mathrm{~b}$, ApJ, submitted

Craig, I. J. D., \& Sneyd, A. D. 1990, ApJ, 357, 653

DeLuca, E. E., \& Craig, I. J. D. 1992 , ApJ, in press

Forbes, T. G., \& Priest, E. R. 1987, Rev. Geophys., 25, 1583

Forbes, T. G., \& Speiser, T. W. 1979, J. Plasma Phys., 21, 107

Hassam, A. B. 1991, Univ. Maryland Plasma Preprint UMLPR 91-046

Imshennik, V. S., \& Syrovatskii, S. I. 1967, Soviet Phys.-JETP, 25, 656

Parker, E. N. 1963, ApJ, 138, 552

1979, Cosmical Magnetic Fields. (Oxford: Clarendon)

\section{REFERENCES}

Petschek, H. E. 1964, in AAS-NASA Symposium on the Physics of Solar Flares(NASA SP-50), 425

Priest, E. R. 1985, Rep. Progr. Phys., 48, 955

Richtmyer, R. D., \& Morton, K. W. 1967, Difference Methods for Initial-Value Problems (2d ed.; New York: Wiley)

Roache, P. J. 1982, Computational Fluid Dynamics (3d ed.; Albuquerque: Hermosa)

Sakai, J. I. 1990, ApJ, 365, 354

Sonnerup, B. U. O. 1973, in Proc. Symp. on High Energy Phenomena on the Sun, (NASA-GSFC Doc. X-693-73-193), 357

Sweet, P. A. 1958, in Electromagnetic Phenomena in Cosmological Physics, ed. B. Lehnert (Cambridge: Cambridge Univ. Press), 123

Vasyliunas, V. M. 1975, Rev. Geophys. Space Phys., 13, 303 